\title{
SOME MAPPINGS WITH TOPOLOGICAL DEGREE ZERO
}

\author{
JANE CRONIN
}

1. Introduction. The existence of solutions of an equation

$$
M(p)=q
$$

is sometimes proved by determining the topological degree of $M$ at $q$. If the degree is different from zero, the equation has a solution. However if the degree is zero, then purely topological considerations give practically no information about solutions. The advantage of the topological degree approach is that comparatively little information about the mapping is needed. In this note we study locally certain mappings which arise in the study of functional equations and whose topological degree can be computed. By taking advantage of working in the small, we obtain sufficient conditions for the existence or nonexistence of solutions if the topological degree of the mapping is zero. Verifying that these conditions hold requires little information beyond that needed to compute the topological degree.

The results we obtain can be applied to obtain existence and nonexistence theorems for the nonlinear integral equations and nonlinear elliptic differential equations studied in [3] and [4].

We study mappings in Euclidean 2-space. Our methods apply to similar mappings in $n$-space $(n>2)$, but if $n>2$ the problem of computing the topological degree or verifying the conditions derived here is hampered by algebraic complications.

2. Lemmas. Consider two mappings, $f$ and $g$, in the Euclidean plane, defined respectively by the pairs of equations:

$$
x_{i}^{\prime}=F_{i}\left(x_{1}, x_{2}\right), \quad i=1,2,
$$

and

$$
x_{i}^{\prime}=G_{i}\left(x_{1}, x_{2}\right), \quad i=1,2,
$$

where $F_{1}, F_{2}, G_{1}$, and $G_{2}$ and their first derivatives are defined and are continuous on an open set containing a closed circle $S$ of radius $r$ and center at the origin. We denote the origin by $\theta$, and we assume that

$$
f^{-1}(\theta)=\theta .
$$

A critical point of mapping $f$ is a point in $f(S)$ which is the image

Received by the editors February 2, 1956. 
under $f$ of a point at which the Jacobian of $F_{1}$ and $F_{2}$ is zero. We suppose that $\theta$ is a critical point of $f$.

Lemma 1. Suppose the mapping

$$
x_{i}^{\prime}=-\left\{G_{i}\left(x_{1}, x_{2}\right)-F_{i}\left(x_{1}, x_{2}\right)\right\}, \quad i=1,2,
$$

takes $S$ into a subsector ${ }^{1} \sigma$ of $S$, and suppose there is an open set $u$ satisfying the following conditions:

(1) $\mathcal{U}$ is connected and simply connected;

(2) there are no critical points of $f$ in $\mathfrak{u}$;

(3) $u \cup[\theta] \supset \sigma$;

(4) $f(S) \supset น$.

Then $\theta \in g(S)$.

Lemma 2. Suppose there is a set $\sigma_{1}$ of the form

$$
\sigma_{1}=\left\{(\rho, \phi) \mid 0<\rho \leqq r, \phi_{3}<\phi<\phi_{4}\right\}
$$

such that $\sigma_{1} \cap f(S)=\varnothing$. Suppose the mapping

$$
x_{i}^{\prime}=-\left\{G_{i}\left(x_{1}, x_{2}\right)-F_{i}\left(x_{1}, x_{2}\right)\right\}, \quad i=1,2 .
$$

takes $S$ into $\sigma_{1}$. Then $\theta \notin g(S)$.

Lemma 2 is trivial. In order to prove Lemma 1 , we let $C$ be a component of $f^{-1}(\mathcal{U})$. Since $\mathcal{U}$ is open and $f$ is continuous, set $C$ is open. Since there are no critical points of $f$ in $\mathcal{U}$, it follows that $f / C$ takes open sets into open sets. Because $u$ is connected, we have: $f(C) \supset \mathcal{u}$. Since $\mathcal{U}$ is simply connected and $C$ is arcwise connected [ 1 , Satz XX, p. 50], it follows by known arguments (see, e.g., [2, proof of Theorem 4.3]) that $f$ is one-to-one on $C$. Hence $f / C$ is a homeomorphism.

It follows directly that $h=f / C \cup[\theta]$ is also a homeomorphism. We write the continuous mapping $h^{-1} / \sigma$ as:

$$
x_{i}=\psi_{i}\left(x_{1}^{\prime}, x_{2}^{\prime}\right), \quad i=1,2,
$$

Now consider the mapping

$$
\begin{aligned}
x_{i}^{\prime \prime}= & -\left\{G_{i}\left[\psi_{i}\left(x_{1}^{\prime}, x_{2}^{\prime},\right), \psi_{2}\left(x_{1}^{\prime}, x_{2}^{\prime}\right)\right]\right. \\
& \left.-F_{i}\left[\psi_{i}\left(x_{1}^{\prime}, x_{2}^{\prime}\right), \psi_{2}\left(x_{1}^{\prime}, x_{2}^{\prime}\right)\right]\right\}, \quad i=1,2,
\end{aligned}
$$

which acts on $\sigma$. This is a continuous mapping of $\sigma$ into itself and hence by the Brouwer fixed point theorem, has a fixed point $\left(x_{10}^{\prime}, x_{20}^{\prime}\right)$. The point

\footnotetext{
1 By a sector of $S$, we mean a set of points $\left\{(\rho, \phi) \mid 0 \leqq \rho \leqq r, \phi_{1} \leqq \phi \leqq \phi_{2}\right\}$ where $\phi_{1}, \phi_{2}$ are given values and $\phi_{2}, \phi_{1}, \phi_{2}-\phi_{1}<2 \pi$. If $r$ is replaced by $r_{1} \leqq r$, we call the resulting set a subsector of $S$.
} 


$$
x_{i 0}=\psi_{i}\left(x_{10}^{\prime}, x_{20}^{\prime}\right), \quad i=1,2,
$$

is mapped into $\theta$ by $g$.

3. Mappings of degree zero. We apply the lemmas to mappings:

$$
L_{\epsilon}:\left(x_{1}, x_{2}\right) \rightarrow\left(x_{1}^{\prime}, x_{2}^{\prime}\right)
$$

defined by the equations:

$x_{i}^{\prime}=P_{m_{i}}\left(x_{1}, x_{2}\right)+\tilde{P}_{m_{i}}\left(x_{1}, x_{2}\right)+H_{i}\left(x_{1}, x_{2}, \epsilon\right), \quad i=1,2,|\epsilon|<\epsilon_{0}$, where $P_{m_{i}}\left(x_{1}, x_{2}\right)$ is a polynomial homogeneous in $x_{1}$ and $x_{2}$ of degree $m_{i} \geqq 2$, and $\widetilde{P}_{m_{i}}\left(x_{1}, x_{2}\right)$ is a term of order exceeding $m_{i}$, i.e.,

$$
\lim _{r \rightarrow 0} \frac{\tilde{P}_{m_{i}}\left(x_{1}, x_{2}\right)}{r^{m_{i}}}=0, \quad\left(r^{2}=x_{1}^{2}+x_{2}^{2}\right),
$$

and $\widetilde{P}_{m_{i}}\left(x_{1}, x_{2}\right)$ has continuous first derivatives. The function $H_{i}\left(x_{1}, x_{2}, \epsilon\right)$ is a continuous function of $\left(x_{1}, x_{2}, \epsilon\right)$ in a neighborhood of $(0,0,0)$, and we assume that $H_{i}\left(x_{1}, x_{2}, \epsilon\right)$ is of order $n_{i}(\geqq 1)$ in $\epsilon$ in the following sense:

$$
H_{i}\left(x_{1}, x_{2}, \epsilon\right)=S_{i}\left(x_{1}, x_{2}\right) \epsilon^{n_{i}}+R_{i}\left(x_{1}, x_{2}, \epsilon\right)
$$

where $S_{i}\left(x_{1}, x_{2}\right)$ is a continuous function of $\left(x_{1}, x_{2}\right) ; \lim _{r \rightarrow 0} S_{i}\left(x_{1}, x_{2}\right)$ $=K_{i}$, a nonzero constant; and

$$
\lim _{\epsilon \rightarrow 0} \frac{R_{i}\left(x_{1}, x_{2}, \epsilon\right)}{\epsilon^{n_{i}}}=0 .
$$

We assume that the topological index at $\theta[1$, p. 470] of the mapping $L$ given by the equations:

$$
x_{i}^{\prime}=P_{m_{i}}\left(x_{1}, x_{2}\right), \quad i=1,2,
$$

is defined. Then if $\epsilon$ is sufficiently small, the topological degree of $L_{\epsilon}$ at $\theta$ relative to a sufficiently small circle with center $\theta$ is, by Rouche's Theorem [1, p. 459], equal to the index of $L$.

Now we use an approach similar to that in $[5, \S 3]$ to determine when Lemmas 1 and 2 can be applied. Since the methods used are always about the same, we indicate just two applications. First we obtain the real linear factors of odd order of $P_{m_{1}}$ and $P_{m_{2}}$ and write:

$$
\begin{aligned}
& P_{m_{1}}\left(x_{1}, x_{2}\right)=p_{1}\left(x_{1}, x_{2}\right)\left[\prod_{j=1}^{r}\left(x_{2}-a_{1 j} x_{1}\right)\right] a_{10} x_{1}, \\
& P_{m_{2}}\left(x_{1}, x_{2}\right)=p_{2}\left(x_{1}, x_{2}\right)\left[\prod_{k=1}^{s}\left(x_{2}-a_{2 k} x_{1}\right)\right],
\end{aligned}
$$


(since the topological index is defined, the factor $x_{1}$ can appear in at most one of the two polynomials $P_{m_{1}}$ and $\left.P_{m_{2}}\right)$ where $p_{i}\left(x_{1}, x_{2}\right)$ is the product of the complex linear factors and the real linear factors with even exponent of $P_{m_{i}}\left(x_{1}, x_{2}\right)$. Since the $p_{i}\left(x_{1}, x_{2}\right)$ never change sign, they do not contribute to the topological index except to determine its sign. The number $a_{10}$ may be chosen so that it is less in magnitude than all the numbers $a_{11}, \cdots, a_{1 r}, a_{21}, \cdots, a_{28}$. Now arrange the numbers $a_{10}, a_{11}, \cdots, a_{1 r}, a_{21}, \cdots, a_{28}$ in order of magnitude and label them by second subscript accordingly. This tells us the order in which $P_{m_{1}}\left(x_{1}, x_{2}\right)$ and $P_{m_{2}}\left(x_{1}, x_{2}\right)$ change sign as one proceeds in a counterclockwise direction along a circle with center at $\theta$ starting from the intersection of the circle with the negative $x_{2}$-axis. Consequently we see at once the value of the topological index except for sign. If the index is different from zero, then the system of equations

$$
L_{\epsilon}\left(x_{1}, x_{2}\right)=0
$$

has a solution. We apply Lemmas 1 and 2 to determine when the system has a solution if the index is zero.

It is easy to see that if there is in the ordered array of numbers $a_{10}, a_{11}, \cdots, a_{1 r}, a_{21}, \cdots, a_{2 s}$ a subset of the form:

$$
a_{1 u}<a_{2 v}<a_{1, u+1}<a_{2, v+1}<a_{1, u+2}
$$

then the set $L(S)$, where $S$ is an arbitrary circle with center at $\theta$, contains a neighborhood of $\theta$. Similarly if such a subset is contained in the array formed if we start from a point on the circle different from its intersection with the negative $x_{2}$-axis (such arrays are obtained from the first array by cyclic permutations), the set $L(S)$ contains a neighborhood of $\theta$. Then the set $L_{0}(S)$ also contains a neighborhood of $\theta$. This last follows from the higher order properties of the $\widetilde{P}_{m_{i}}$, i.e., we apply the fact that if $r$ is sufficiently small, then

$$
\left|\tilde{P}_{m_{i}}\right|<A_{i} r^{m_{i}}
$$

where $A_{i}$ is an arbitrarily small positive constant.

The critical points of $L$ are the images of the finite set of lines through $\theta$ on which the Jacobian of $P_{m_{1}}$ and $P_{m_{2}}$ vanishes. (If the Jacobian vanishes identically, then either the topological index of $L$ is not defined or $P_{m_{1}}$ and $P_{m_{2}}$ have no real linear factors.)

THEOREM 1. If there is a subset of form (1) in the array and if for sufficiently small positive $\epsilon$, the points

$$
\left(-K_{1} \epsilon^{n_{1}},-K_{2} \epsilon^{n_{2}}\right)
$$

are not critical points of $L$, then corresponding to any sufficiently small 
circle $S$ with center at $\theta$, there is an $\epsilon_{0}>0$ such that if $0<\epsilon<\epsilon_{0}$, then $\theta \in L_{\epsilon}(S)$.

Proof. From the continuity and higher order properties of the $\widetilde{P}_{m_{i}}$, it follows that there are positive numbers $r_{1}$ and $\epsilon\left(r_{1}\right)$ such that if $S_{1}$, a circle with center $\theta$, is of radius $r_{1}$, there is a sector $\sigma$ of $S_{1}$ such that $L_{0}$ has no critical points in an open set

$$
\mathcal{U}_{0}=\left\{(\rho, \phi) / 0<\rho<r_{0}, \phi_{5}<\phi<\phi_{6}\right\}, \quad\left(r_{0}>r_{1}\right)
$$

which contains $\sigma-\theta$. Also for $0<\epsilon<\epsilon_{1}\left(r_{1}\right)$, the points

$$
\left(-K_{1} \epsilon^{n_{1}},-K_{2} \epsilon^{n_{2}}\right)
$$

are in the interior of $\sigma$. There is a circle $S_{2}$ with center $\theta$ of maximum radius $r_{2}$ such that $S_{2} \subset L_{0}\left(S_{1}\right)$, and $\epsilon_{1}\left(r_{1}\right)$ can be chosen so that if $0<\epsilon<\epsilon_{1}\left(r_{1}\right)$, the points

$$
\left(-K_{1} \epsilon^{n_{1}},-K \epsilon^{n_{2}}\right)
$$

are in the interior of $\sigma \cap S_{2}$. Now positive constants $B_{1}(r), B_{2}\left(r_{2}\right)$, and $\epsilon_{2}<\epsilon_{1}\left(r_{1}\right)$ may be chosen such that if $\left|\xi_{1}\right|<B_{1}\left(r_{2}\right),\left|\xi_{2}\right|<B_{2}\left(r_{2}\right)$, $0<\epsilon<\epsilon_{2}$, the points

$$
\left(-\left(K_{1}+\xi_{1}\right) \epsilon^{n_{1}},-\left(K_{2}+\xi_{2}\right) \epsilon^{n_{2}}\right)
$$

are in the interior of $\sigma \cap S_{2}$.

Hence there is a circle $S_{3}$ with center $\theta$ and radius $r_{3}<r_{1}$ and an $\epsilon_{3} \leqq \epsilon_{2}$ such that if $\left(x_{1}, x_{2}\right) \in S_{3}$ and $\epsilon<\epsilon_{3}$, then

$$
\left(-H_{1}\left(x_{1}, x_{2}, \epsilon\right),-H_{2}\left(x_{1}, x_{2}, \epsilon\right)\right)=\left(-\left(K_{1}+\xi_{1}\right) \epsilon^{n_{1}},-\left(K_{2}+\xi_{2}\right) \epsilon^{n_{2}}\right)
$$

is in $\sigma \cap S_{2}$. Choose circles $S_{4}, S_{5}$, both with center $\theta$, such that $S_{5} \Phi S_{4} \subset L_{0}\left(S_{3}\right)$. Choose $\epsilon_{4}$ so that for $0<\epsilon<\epsilon_{4}$ and $\left(x_{1}, x_{2}\right) \in S_{3}$,

$$
\left(-\left(K_{1}+\xi_{1}\right) \epsilon^{n_{1}},-\left(K_{2}+\xi_{2}\right) \epsilon^{n_{2}}\right) \in \sigma \cap S_{5} .
$$

Now apply Lemma 1 letting the mappings $f$ and $g$ be $L_{0}$ and $L_{\epsilon}$ where $0<\epsilon<\epsilon_{4}$ and letting $S$ be the circle $S_{3}$ above and the open set $u$ be $u_{0} \cap$ (Interior $S_{4}$ ).

The theorem can be sharpened if we investigate the real linear factors of odd order of the Jacobian $J\left(P_{m_{1}}, P_{m_{2}}\right)$ of $P_{m_{1}}$ and $P_{m_{2}}$. We write:

$$
J\left(P_{m_{1}}, P_{m_{2}}\right)=q\left(x_{1}, x_{2}\right)\left[\prod_{x=l}^{t}\left(x_{2}-a_{3 l} x_{1}\right)\right] a_{30} x_{1}
$$

where $q\left(x_{1}, x_{2}\right)$ is the product of the complex linear factors and the real linear factors with even exponent. (If the factor $x_{1}$ appears, we may 
choose $a_{30}$ equal to $a_{10}$ and so that it is less in magnitude than all the numbers $a_{11}, \cdots, a_{1 r}, a_{21}, \cdots, a_{2 s}, a_{31}, \cdots, a_{3 t}$ )

We arrange the numbers $a_{10}, a_{11}, \cdots, a_{1 r}, a_{21}, \cdots, a_{2 s}, a_{30}$, $a_{31}, \cdots, a_{3 t}$ in order of magnitude and label them by second subscript accordingly. This array shows the order in which $P_{m_{1}}$ and $P_{m_{2}}$ change sign and also shows the order of the points at which the image of the circle "turns around," i.e., the points where the Jacobian changes sign. Now suppose there is in this array a subset of the form:

$$
a_{3 w}<a_{1 u}<a_{2 v}<a_{1, u+1}<a_{2, v+1}<a_{3, w+1} .
$$

Let $\lambda_{0}$ and $\lambda_{1}$ be the half-rays in the region $x_{1}>0$ which are contained in the lines:

$$
x_{2}-a_{3 w} x_{1}=0
$$

and

$$
x_{2}-a_{3, w+1} x_{1}=0
$$

respectively. Assume that the slope of the tangent at the origin of the curve $L\left(\lambda_{0}\right)$ is less than the slope of the tangent at the origin of the curve $L\left(\lambda_{1}\right)$. Then the set $L(S)$, where $S$ is an arbitrary circle with center at $\theta$, contains a neighborhood of $\theta$ and consequently $L_{0}(S)$ also contains a neighborhood of $\theta$. Thus we may replace in Theorem 1 the hypothesis that there is a subset of the form (1) with the hypothesis that there is a subset of form (2). (As before, we include in this hypothesis the possibility that such a subset is contained in the array obtained if we start from a point on the circle different from its intersection with the negative $x_{2}$-axis.)

Now if $P_{m_{1}}$ or $P_{m_{2}}$ has no real linear factors, there is a quadrant $Q$ of the $x_{1} x_{2}$-plane which does not intersect $L(S)$ except at the origin. Also, returning to the array of numbers $a_{10}, a_{11}, \cdots, a_{1 r}, a_{21}, \cdots, a_{2 s}$, if we assume that the array has the form:

$$
a_{10}<a_{11}<a_{21}<a_{22}<a_{12}<a_{13}<\cdots,
$$

i.e., roots of the same polynomial occur in pairs, there is again a quadrant $Q$ which does not intersect $L(S)$ except at the origin.

THEOREM 2. If one of the polynomials $P_{m_{1}}, P_{m_{2}}$ has no real linear factors or if the array of roots is of the form (3) and if for sufficiently small positive $\epsilon$, the points

$$
\left(-K_{1} \epsilon^{n_{1}},-K_{2} \epsilon^{n_{2}}\right)
$$

are in the interior of $Q$, then corresponding to any sufficiently small 
circle $S$ with center $\theta$, there is an $\epsilon_{0}>0$ such that if $0<\epsilon<\epsilon_{0}$, then $\theta \notin L_{\epsilon}(S)$.

Proof. This is an application of Lemma 2. The same type of argument as in the proof of Theorem 1 is applied.

If $\widetilde{P}_{m_{i}}\left(x_{1}, x_{2}\right)$ is a sum or infinite series of homogeneous polynomials, the methods used here may also be applied in the more complicated case where the topological index of $L$ is not defined but the topological index at $\theta$ of the mapping

$$
x_{i}^{\prime}=P_{m_{i}}\left(x_{1}, x_{2}\right)+\tilde{P}_{m_{i}}\left(x_{1}, x_{2}\right), \quad i=1,2,
$$

is defined. We must then consider instead of mapping $L$, a mapping

$$
x_{i}^{\prime}=\sum_{q=0}^{r_{i}} P_{m_{i}+q}\left(x_{1}, x_{2}\right), \quad i=1,2,
$$

where the $P_{m_{i}+q}\left(x_{1}, x_{2}\right)$ are homogeneous polynomials of degree $m_{i}+q$. Some results can be obtained, but the computations are so much more complicated that it seems practical to work directly with each given mapping rather than attempt to obtain general theorems.

\section{BIBLIOGRAPHY}

1. P. Alexandroff and H. Hopf, Topologie 1, Berlin, 1935 (reprinted Ann Arbor, Mich., Edwards Brothers.)

2. R. G. Bartle, Implicit functions and solutions of equations in groups, Math. Zeit. vol. 62 (1955) pp. 335-346.

3. J. Cronin, Branch points of solutions of equations in Banach space, Trans. Amer. Math. Soc. vol. 69 (1950) pp. 208-231.

4. - Branch points of solutions of equations in Banach space II, Trans. Amer. Math. Soc. vol. 76 (1954) pp. 207-222.

5. - Topological degree of some mappings, Proc. Amer. Math. Soc. vol. 5 (1954) pp. 175-178.

SOUTHBRIDGe, Mass. 